Arqueología y Sociedad,

№ 22, 2010

\title{
MANEJO DEL ESPACIO EN EL SITIO ARQUEOLÓGICO DE HUANCHOSMARCA DURANTE EL PERIODO INTERMEDIO TARDÍO
}

\author{
Milton Luján Dávila*
}

\section{Resumen}

La caracterización a la problemática del manejo y uso del espacio para sitios de altura de la cuenca Alta del Chillón han sido estudiadas de manera sucinta, el presente artículo propone correlacionar los datos de campo llevados a cabo en 1998 con diversos estudios de carácter arqueológico y etnohistórico para el área de estudio, bajo esta perspectiva se plantea que el sitio de Huanchosmarca fue construido bajo un modelo de crecimiento asimétrico relacionado de manera directa a un espacio sagrado cerro Cahuara «apu tutelar» de esta área, sitio que estuvo vinculado a la parcialidad de Viscas, ayllu encargado de los poblados de Huanchosmarca y Quecamarca.

\section{Palabras clave}

Manejo del espacio, cuenca alta del Chillón, Huanchosmarca.

\begin{abstract}
While the management and use of space at sites in the high altitude basin of Chillón have been studied succinctly, this article proposes to correlate field data conducted in 1998 with several diverse archaeological and ethnohistorical studies for the area. With this perspective we suggest that the site of Huanchosmarca was constructed under a model of asymmetrical growth in a manner directed at the sacred space of Cerro Cahuara or "guardian $a p u$ " of this area, a place that was linked to the parcialidad Viscas, the ayllu responsible for the Huanchosmarca and Quecamarca villages.
\end{abstract}

\section{Keywords}

Management and use of space, altitude basin of Chillón, Huanchosmarca.

* Codirector del PIA [ParaCo] Cochayoc, Región Ancash, Director del PIA Huacosmarca, Cuenca Alta del Chillón, Canta.

Correo electrónico: miltonrld@hotmail.com; miltonrld@gmail.com 


\section{INTRODUCCIÓN}

El estudio llevado a cabo en el sitio arqueológico de Huanchosmarca en el anexo de San Lorenzo, provincia de Canta; sugiere una propuesta de análisis espacial por guardar relaciones estrechas de uso, manejo del espacio y arquitectura, al igual que otros sitios instalados en altura en las dos márgenes de la Cuenca Alta del río Chillón durante el Periodo Intermedio Tardío.

Manejo espacial que habría alcanzado niveles de articulación entre asentamiento y su entono paisajístico, caracterización de suma importancia para explicar niveles de desarrollo arquitectónico que se vieron inversamente relacionados entre sitios como: Viscas, Queca y Huanchos en la margen izquierda del río Chillón. Donde los niveles de correspondencia al interior permiten proponer tipos de actividad domestica y ritual en espacios intramuros y extramuros, siendo caso de estudio el sitio de Huanchos. Sin embargo, es necesario establecer que los datos obtenidos son resultado de trabajos de campo que permiten correlacionar aspectos políticos, sociales y económicos relacionados a datos etnohistóricos para los grupos étnicos instalados en zonas Yunga y Quechua del valle del Chillón.

Murra $(1975,2009)$ advirtió que procesos de interacción entre pueblos de altura y valle medio se dieron a través de un «control vertical de un máximo de pisos ecológicos», al cual se adicionaron elementos articuladores de fortalecimiento, a través de la existencia de dominios territoriales aptos para la agricultura como lo sugirió Rostworowski (1978) y cooperación propuesta por Dillehay (1977), además de procesos políticos y económicos que alcanzaron vínculos de reciprocidad y redistribución asociados a posibles elementos de interacción que quizá no se pudieron haber amoldado a conexiones directas a núcleos principales, creando así posibles flexibilidades de carácter vertical en esta área (Kaulicke 1974-1975).

Procesos que aun son necesarios esclarecer, debido a la existencia de interacciones horizon- tales entre asentamientos de la sierra de Lima durante el Periodo Intermedio Tardío, basados en el uso y manejo del espacio, patrón arquitectónico a nivel intramuro y extramuro en diversos asentamientos relacionados directamente a concepciones religiosas a espacios naturales -sagrados-.

En este sentido, las investigaciones desarrolladas en Huanchosmarca llevan a proponer rasgos emparentados entre arquitectura y distribución de las unidades arquitectónicas que fueron manejadas de manera asimétrica

\section{LINEAMIENTO POLÍTICO Y SOCIAL DEL ÁREA DE ESTUDIO}

La organización sociopolítica en la cuenca Alta del Chillón durante el Periodo Intermedio Tardío se habría establecido a través de intensos procesos de intercambio y reciprocidad que implicó marcadas influencias en muchas de estas poblaciones. En este escenario, las interacciones económicas y políticas sugerida por Dillehay (1976) y Silva (1996), cultural y religiosa propuesta de manera implícita por Ávila (2009[i1598?]) entre pueblos yungas y quechuas, habría establecido dos tipos de control, una de carácter vertical como se ha mencionado y consecuentemente otra de carácter horizontal en dicha área.

De acuerdo al modelo de carácter vertical se habrían dado intercambios de productos alimenticios y bienes manufacturados en épocas de paz entre las poblaciones instaladas en estas zonas, generando relaciones verticales entre estos pueblos, creándose de esta manera interacciones forzadas en un máximo de pisos ecológicos (Murra 1977, 2009, Rostworowski 1978, Dillehay 1987, Marcus \& Silva 1988, Farfán 1995, Netherly 1998) producidos no solo por necesidades primarias o por la obtención de productos tales como la coca, ají, maní, yuca, etc. sino a su vez habrían provocado conflictos, como fue el caso entre los Canta y Colli por un afán de controlar territorios productivos antes de la ocupación Inca (Rostworowski 1978). 
Si bien, el curacazgo de Canta manejó ayllus de manera directa a través de una economía móvil y redistributiva por la obtención de recursos de diferentes pisos ecológicos, el estilo arquitectónico se dio de manera particular al interior de unidades distribuidas de manera asimétrica. Cabe la posibilidad entonces que se hubiera producido relaciones de carácter cooperativopacifico entre los serranos y yungas en el valle intermedio (Dillehay 1987: 425) en épocas de paz permitiendo que los Colli y los Canta hayan tenido estrechas relaciones, pero que no necesariamente implicará vínculos directos entre estos grupos culturales como lo establece Dillehay, por cuanto problemas de litigio y control de tierras se dieron desde el Periodo Intermedio Tardío.

Bajo este modelo de carácter diacrónico para el área del Chillón durante las últimas épocas se habría producido a su vez coyunturas creadas por grupos étnicos de valle medio y alto que habrían generado abastecimientos en las economías de estos pueblos (Fig. 1). Sin embargo, aun no parece quedar claro la naturaleza de estas relaciones entre grupos culturales de la parte alta (Fig. 2), sobre todo cuando se trató de zonas de explotación que no tuvieron conexión directa a núcleos principales de asentamientos con poder político, generadores de flexibilidades verticales que encontraron sus límites y expansiones en períodos de mayor fuerza socio-económico y, que habrían alcanzado interacciones en forma horizontal (Kaulicke 1975: 30), hecho que podría ser explicado en base a concepciones religiosas dada la presencia de espacios sagrados de altura vinculadas de manera directa a sitios con rasgos arquitectónicos particulares, las cuales se vincularon a dioses -apus tulerares, cochas- (ver Luján \& Sánchez 1999) e -ídolos(ver Luján 2009).

En este sentido, en este espacio se sugiere vínculos a través de restos tangibles y cultura material relacionados a elementos simbólicos que llevará a estrechar interacciones de carácter horizontal entre grupos étnicos asentados en la parte alta de la sierra de Lima (id. 2009), evidencias que fortalecerían aún más el manejo de nuestras variables, y así poder acercarnos a una explicación mayor del área de estudio.

\section{Problemática del Área de estudio}

Consolidado el desarrollo de los diversos Señoríos durante el Periodo Intermedio Tardío entre c. 1000 y 1430 d.C., con áreas territoriales no definidas, se sugiere una suerte de influencias culturales en áreas de frontera. Al respecto Dillehay y Netherly (1998) proponen que las fronteras alcanzadas durante la expansión Inca estuvieron vinculadas a una diversidad de situaciones y relaciones durante la ocupación de sociedades culturalmente distintas venidas con anterioridad. Es decir, cada grupo étnico estuvo establecido fuertemente dentro de su área de desarrollo político, económico, cultural e ideológico (Fig. 2). Esto advertiría entonces que poblamientos de mayor jerarquía con asentamientos principales controlaran sitios que pasaran a formar parte de un Señorío o Curacazgo, debido a que no contaban con áreas definidas regionalmente, en la cual se desarrollaron múltiples actividades de acuerdo a la complejidad de sus organizaciones internas y desarrollo económico-productivo tal como lo establecen Parsons y Matos (1977) o Earle, D'altroy y Leblanc (1978) para la sierra central o con características religiosas como lo propone Luján (2009) para la parte alta de Canta ${ }^{1}$.

De esta forma, se han venido manejado problemas de territorialidad en cuanto a Curacazgos, que habrían deslindado sus límites a través de canales, ríos, cerros (Rostworowski 1978) o acequias y canales principales que se desprendían a través de numerosos ramales que daban pie al riego de sus tierras de los diversos sitios

1 Durante los trabajos de investigación desarrollado en Huacosmarca 2009 en la margen derecha del río Chillón, se ha confirmado que el sitio representó un centro de culto al agua para diversas poblaciones asentadas en las dos márgenes, área en la que se identificó un ídolo en la parte más alta del sitio asociado a un conjunto de ofrendas de alta representación, donde a su vez las unidades arquitectónicas parecen representar de manera significativa a cada comunidad durante el Periodo Intermedio Tardío (ver Luján 2009). 


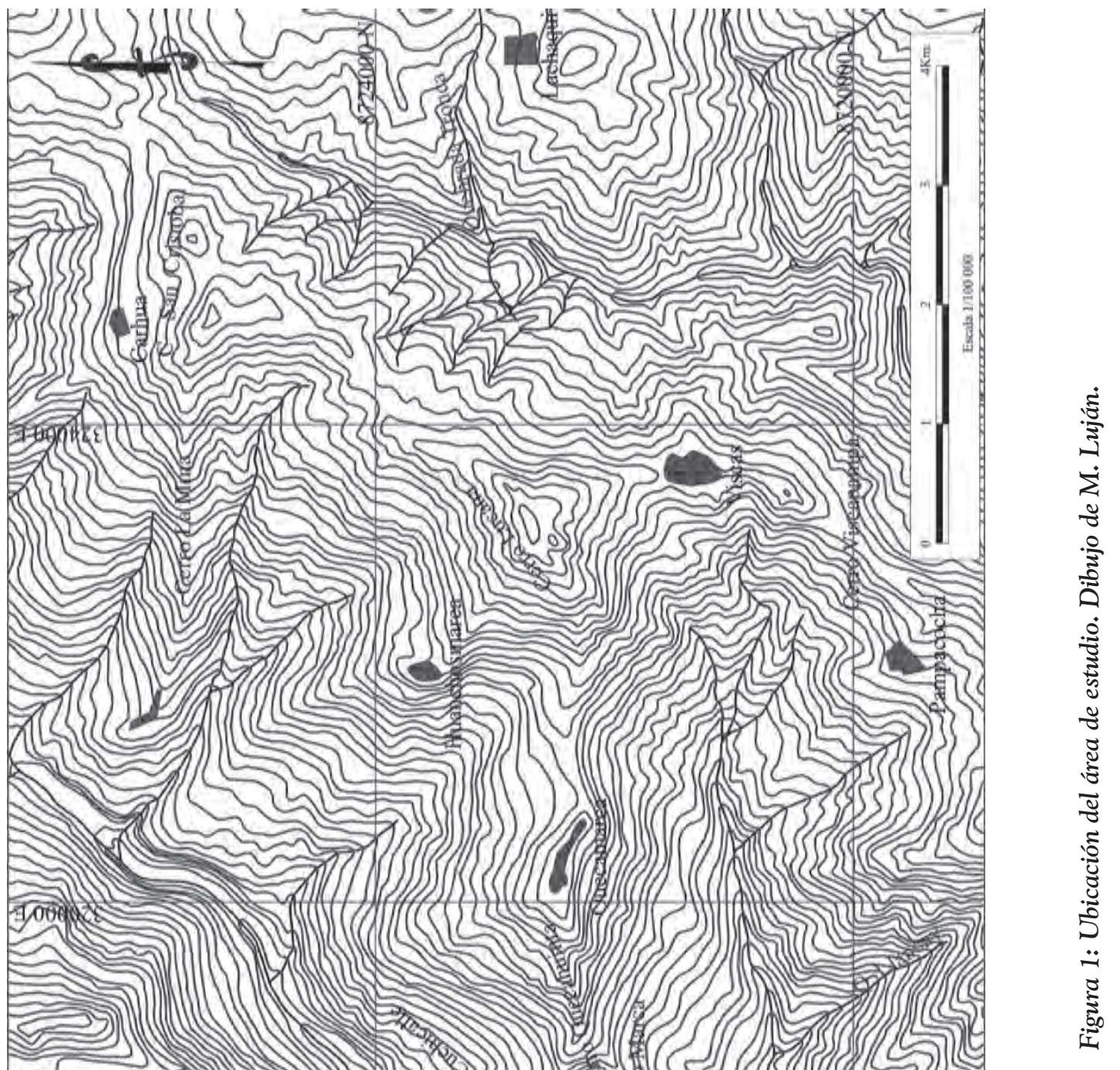




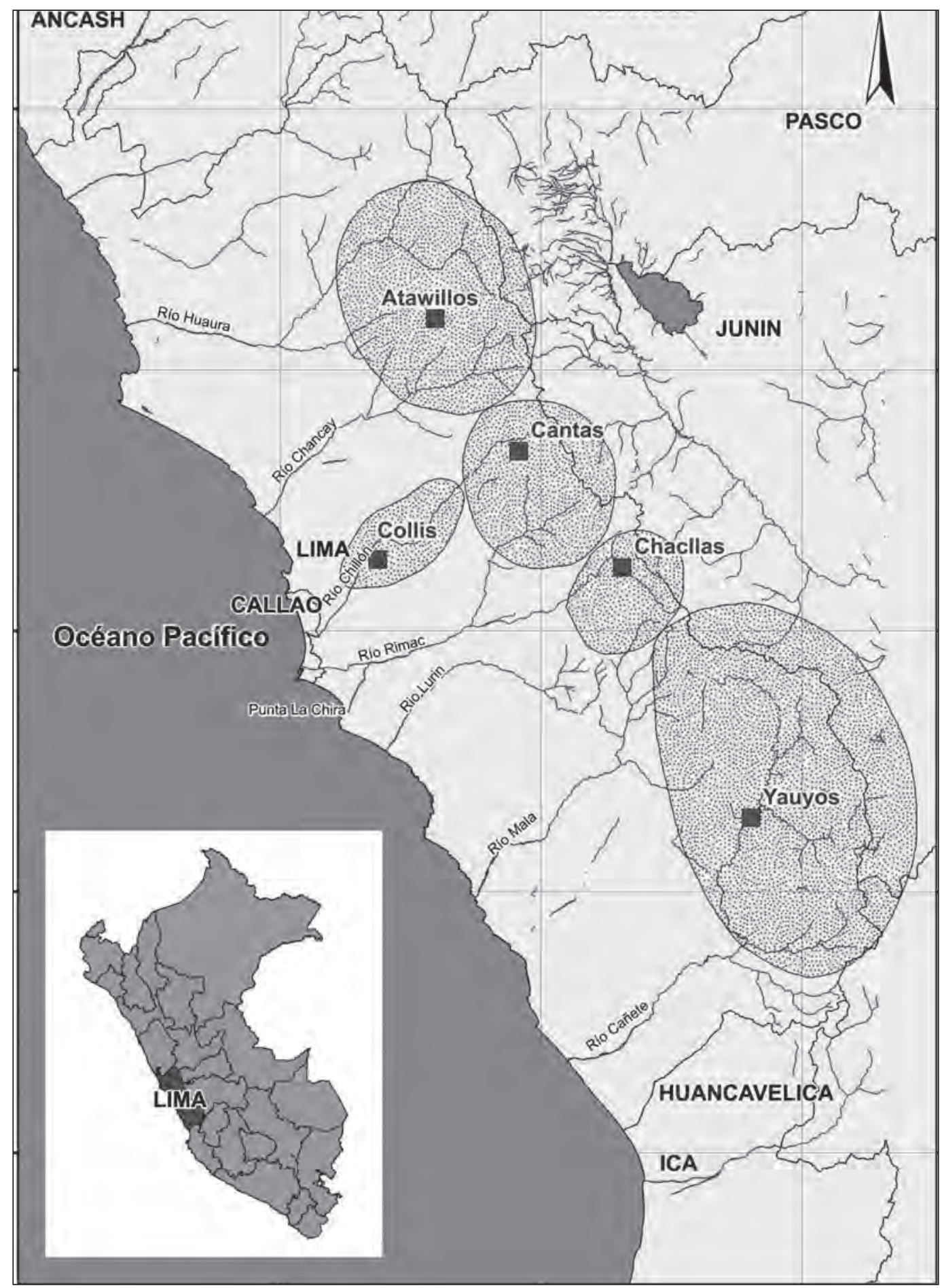

Figura 2: Área cultural de los curacazgos de la sierra de Lima Dibujo R. Martínez. 
de altura durante el Periodo Intermedio Tardío, que fueron fortalecidos en el Horizonte Tardío.

Tema, que aún es incierto para el caso del Curacazgo de Canta que viene siendo estudiado por Farfán desde la década de los 80 de fines del siglo pasado, asentamiento que estuvo conformado por ocho Ayllus, todas situadas en la margen izquierda del río Chillón, ubicados en varios pisos ecológicos desde la Chaupiyunga hasta la Puna.

Para la parte alta, -margen izquierda del río Chillón- Rostworowski (1978) establece que en la segunda visita hecha por los clérigos Fray Antonio de Figueroa y Gómez Hernández en 1553, deslindaron para el área de Canta siete parcialidades, las cuales estuvieron conformados por los Canta, Locha, Carua, Visca, Lachaqui, Copa y Esquibamba, a excepción de Caxa Uri, que no fue mencionado por los clérigos.

Ya que la parcialidad de Viscas habría correspondido en aquel entonces a un ayllu menor del curacazgo de Canta, de mayor importancia que los asentamientos de Queca y Huanchos tributarios de Viscas (Fig.1), que llego a contar con 118 casas habitadas, 80 despobladas y 18 depósitos bajo el cargo de un Señor Principal y dos encargados o jefes locales de menor jerarquía (Rostworowski 1978: 164), autoridades que habrían estado relacionados a los poblados mencionados a fines del siglo XVI y principios del siglo XVII.

Sitios: política, económica y culturalmente establecidos bajo influencia del curacazgo de Canta, que consolidó su poder hacia sus ayllus menores a través de la cultura material (cerámica, textil, etc.), arquitectura, costumbres y religiosidad vinculado a un centro de culto instalado en la parte alta margen derecha del río Chillón (Ávila 2009[i1598?]: 79) que estaría relacionado al sitio de Huacosmarca (Luján 2009).

Un caso particular para explicar y establecer estos vínculos sería través del tipo de asentamientos ubicados en la sierra de Lima, donde hubo aprovechamiento al máximo de la geomorfología condicionada a espacios agrestes, que los llevó a elaborar edificios bajo una adecuada técnica constructiva con rasgos arquitectónicos particulares para el área a través de cámaras, hornacinas, vanos de acceso, con espacios mayores como: patios, plazas con huancas en algunos casos (Farfán 1993) y elementos ofrendatorios relacionados a espacios sagrados «Apus o Camaquenes» (Huertas 1981).

\section{Caracterización Del PRoblema}

La problemática del manejo de espacio y la arquitectura de los asentamientos instalados en la sierra de Lima vinculados a espacios sagrados, en este sentido faltan ser esclarecidos como parte de procesos de desarrollo alcanzados por grupos culturales como los Atavillos y Yauyos asentados hacia los dos extremos de la vertiente del río Caraguaillo hoy rio Chillón (Fig. 2), asentamientos que desarrollaron crecimientos asimétricos de orden espacial con poblaciones vinculados horizontalmente, de ahí que puedan relacionarse como es el caso de Chiprak con Rupak, Pumacoto, Huishco, etc. (Farfán 1995); Cantamarca con Viscas, Queca, Huanchos etc. (Luján \& Sánchez 1999); Los Yauyos con los Chacllas, Carampoma, etc. (Cornejo 2000) generadores de contactos culturales entre diversos curacazgos y ayllus menores en la sierra de Lima (Ministerio de Fomento 1881: 77) instalados desde los 2400 hasta los 4200 metros sobre el nivel del mar.

De esta manera, el crecimiento asimétrico de estos asentamientos sugiere haber tenido vínculos de religiosidad a espacios sagrados «naturales», en la que se desarrollaron ceremonias y ritos de veneración y culto al agua (Luján 2009), hecho que habría permitido consolidar a los dioses de la sierra de Lima, que a principios del siglo XVII aún existían en la provincia de Canta, Chancay y Cajatambo, bajo un cuerpo jerárquico de sacerdotes indígenas encargados de mantener el culto a ídolos y mallquis, que impidieron que desaparezca el culto al Camaquen «Dios Hacedor» (Huertas 1981: 54). Permitido asimismo 
la instalación de sitios asociados a la naturaleza de altares ancestrales como lo establece Schjellerup (1992) para el área de Huánuco y Luján (2009) para el área de Canta. En este sentido, el concepto de religiosidad se entendería en cierta forma como algo diversificado, pero a la vez identificado al interior de un espacio territorial con costumbres ancestrales reflejadas en usos de dialectos, para el caso de la sierra de Lima el «cauqui» (Villar Córdoba 1935, Favre 1983) hablado posiblemente hasta épocas muy tardías y pérdidas durante la Colonia. Dato etnográfico que daría sustento a interacciones de diferente orden que los llevo por estrechas relaciones de identidad cultural e ideológica, condicionante que permitiría esclarecer vínculos existentes en la cuenca Alta del Chillón como por v.g., en el ayllu de Viscas, al que se vinculará los poblados de Huanchos y Queca ubicadas en la margen izquierda del río Chillón ubicados por sobre los 3200 metros sobre el nivel del mar.

De esta manera la hipótesis del uso y función de los espacios de asentamientos con crecimiento asimétrico vinculados a elementos de carácter religioso asociado a espacios naturales sacralizados establecerían relaciones directas a procesos de productividad, espacios donde se dieron rituales y ceremonias sagradas como fue el caso del cerro Lucana o Cahuara de difícil acceso, vinculado a los poblados prehispánicos de Viscas, Queca y Huanchos.

Planteamiento que puede establecerse a partir de los documentos, del cual Huertas (1981: 54) establece que:

«A mediados del siglo XVII, en Cajatambo se hacían ceremonias antes de ser construidas las casas, los ritos constituían en matar una llama, ofrendar con coca y chicha e invitaban a un sacerdote indígena o chaman para que ofrecieran el ritual, personaje que regaba con sangre y molle el área donde iba ser construida la vivienda, invocando a ídolos y mallquis para que las casas se mantuvieran incólumes frente a los embates del tiempo».
Cita, que sugiere la posibilidad que los rasgos arquitectónicos tuvieron mayor probabilidad de difundirse ya sea por migraciones, conquistas militares o imposiciones religiosas, tal como lo propone Raffino (1991) para grupos culturales del noroeste Argentino y, que podrían ser tomados en consideración para el caso de esta área. Lo que lleva a plantearnos en este sentido que los diversos grupos se motivarán por edificar sus sitios en función a espacios sagrados, para lo cual utilizaron como materia constructiva la piedra de cerro cortada y/o trabajada para edificar sus recintos, conformados por vanos de acceso e ingresos hacia las cámaras asociados a ofrendas (Fig. 8).

Donde el manejo espacial y temporal estuvo relacionado al dato constructivo, lo que lleva a proponer además que se «[...] procreo una diversificación regional de estilos arquitectónicos en diferentes áreas como segmentación de los estadios evolutivos de la cultura y con una segunda alternativa concerniente a la creciente complejización de la vivienda familiar[...]» ( Id. 1991), modelo de crecimiento asimétrico que pudo darse en la cuenca alta del Chillón, que implicará procesos de concentración demográfica creciente y nuevas respuestas tecno-económicas a problemas que está demando y que se encuentra diferenciadas entre la arquitectura de los Atawillos, Cantas y Yauyos, etc., en la sierra de Lima.

Sistematización que permite deducir el avance y desarrollo del manejo no solo constructivo sino del espacio, recreado en la naturaleza propia de los individuos que fueron controlados por señores principales o curacas, quienes mandaron construir grandes o pequeños asentamientos con sus propias complejidades, como pudo ser el caso de Huanchos, poblado directamente relacionado a la parcialidad de Viscas dependiente del curacazgo de Canta, que presenta un modelo asimétrico de crecimiento adaptado a la morfología del cerro que se encuentra en dirección Norte, conformado al interior por unidades de vivienda que permitieron relaciones directas de vida familiar y comunal denominada como uni- 


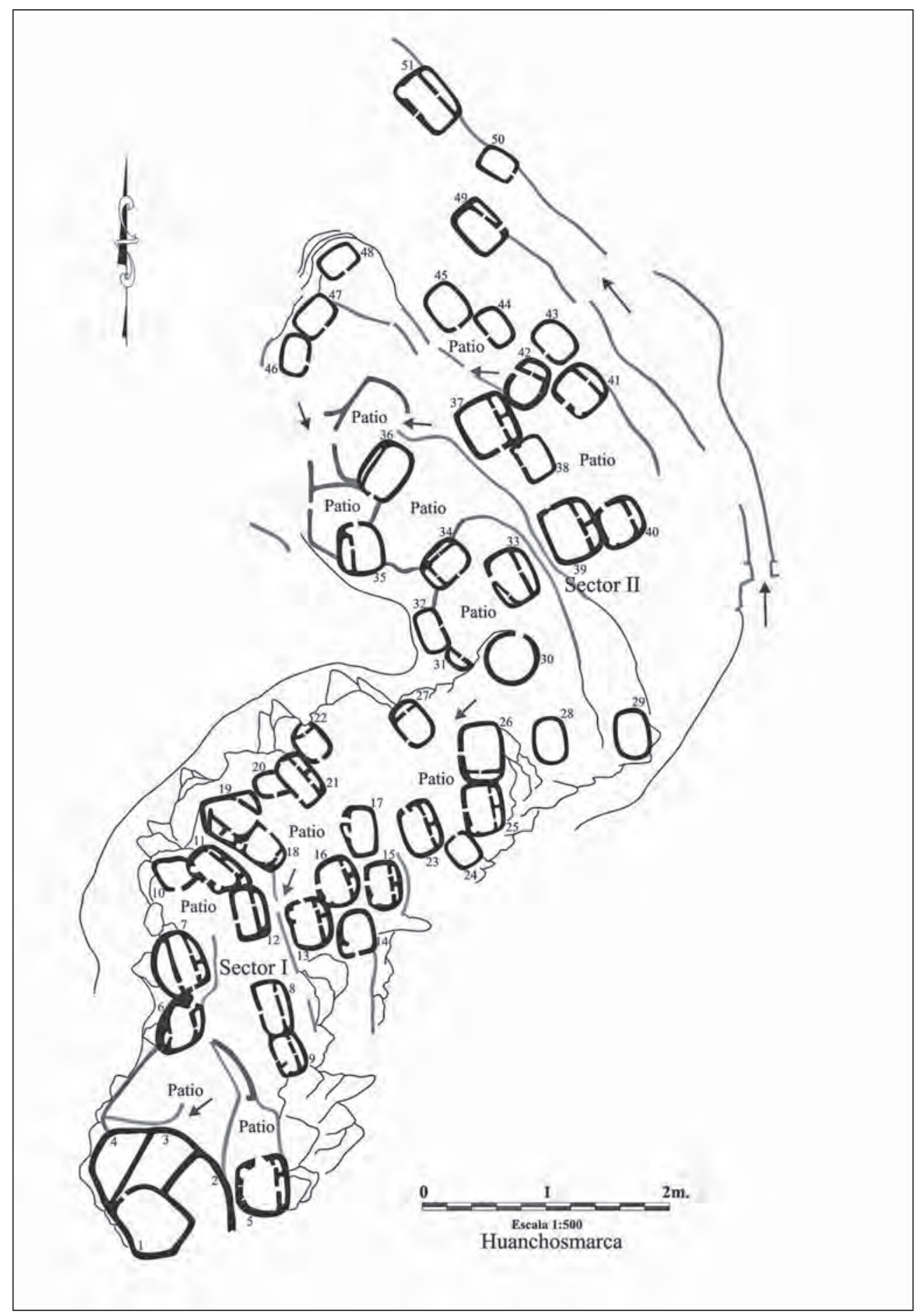

Figura 3: Plano general de Huanchosmarca. Dibujo M. Luján. 


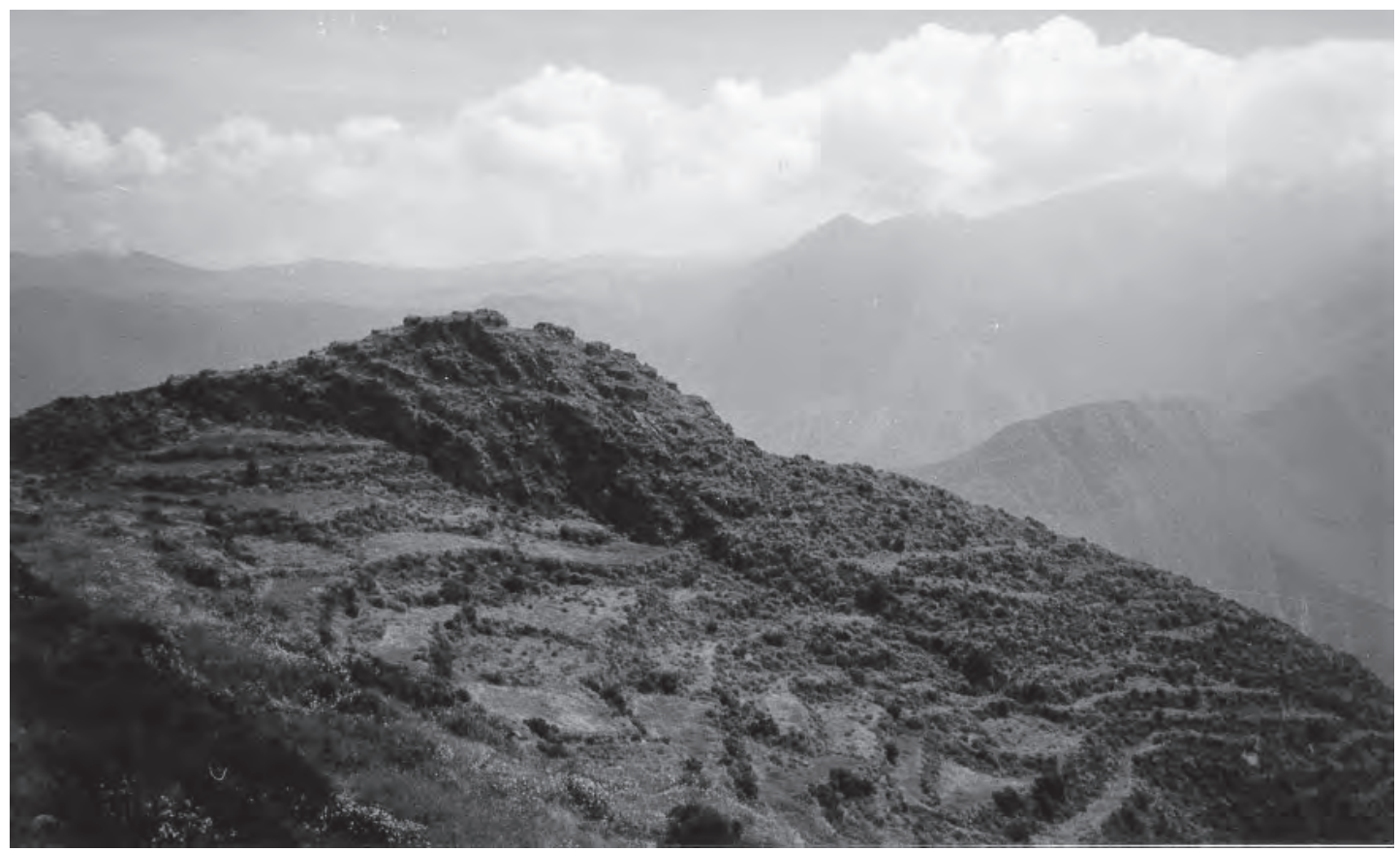

Figura 4: Ubicación del sitio sobre la cresta de un cerro. Fotografía M. Luján.

dades nucleadas (Thompson 1972, Parsons \& Matos 1977, Schjellerup 1992, Bonnier 1997) o unidades alveolares (Lavalleé y Julien 1983) o unidades familiares (Farfán 1988). (Fig. 3).

Definiciones que no llevan a contradicciones, sino mas bien a establecer diferenciaciones posiblemente de carácter estructural, para el caso de estudio optada por unidades familiares como modelo que se conceptúa para el entendimiento de una relación familiar o comunal, mientras que la primera definición habría cumplido funciones quizá un tanto complejas debido a territorios geográficos más complejos. Sin embargo, estos conceptos podrían esclarecer sistemas diversos de organización al interior de los asentamientos.

En este panorama, Huanchos y Queca como parte de la parcialidad de Viscas esclarecen el manejo del espacio y función relacionada de manera directa como asentamientos que conceptúan aspectos culturales y religiosos, bajo crecimientos irregulares con una categoría funcional organizada y articulada y, no como asentamientos desorganizados y desarticulados sugeridos por Agurto (1989).

Se podría deducir entonces para el caso de Huanchosmarca (Fig. 3, 4) que el sitio adoptó un patrón y manejo espacial propio del Periodo Intermedio Tardío, con características similares a otros asentamientos de altura, con ciertas diferenciaciones al interior o área intramuro, teniendo como ejemplo una arquitectura con recintos constructivamente muy bien elaborados, que los llevo a recrear su espacio interior con sistemas de pasajes (Fig. 3, 5), rampas naturales, gradas, patios asociadas a las viviendas, depósitos y posibles corrales (ver fig. 3) que habrían cumplido diferentes funciones sean de carácter doméstico, laboral y ritual en zonas de altura del área Andina.

\section{Manejo del espacio}

Los asentamientos de mayor envergadura con crecimiento asimétrico como Rupak, Cantamarca, Naupahuasi, etc.; se magnificaron por la solides constructiva y buen manejo del es- 


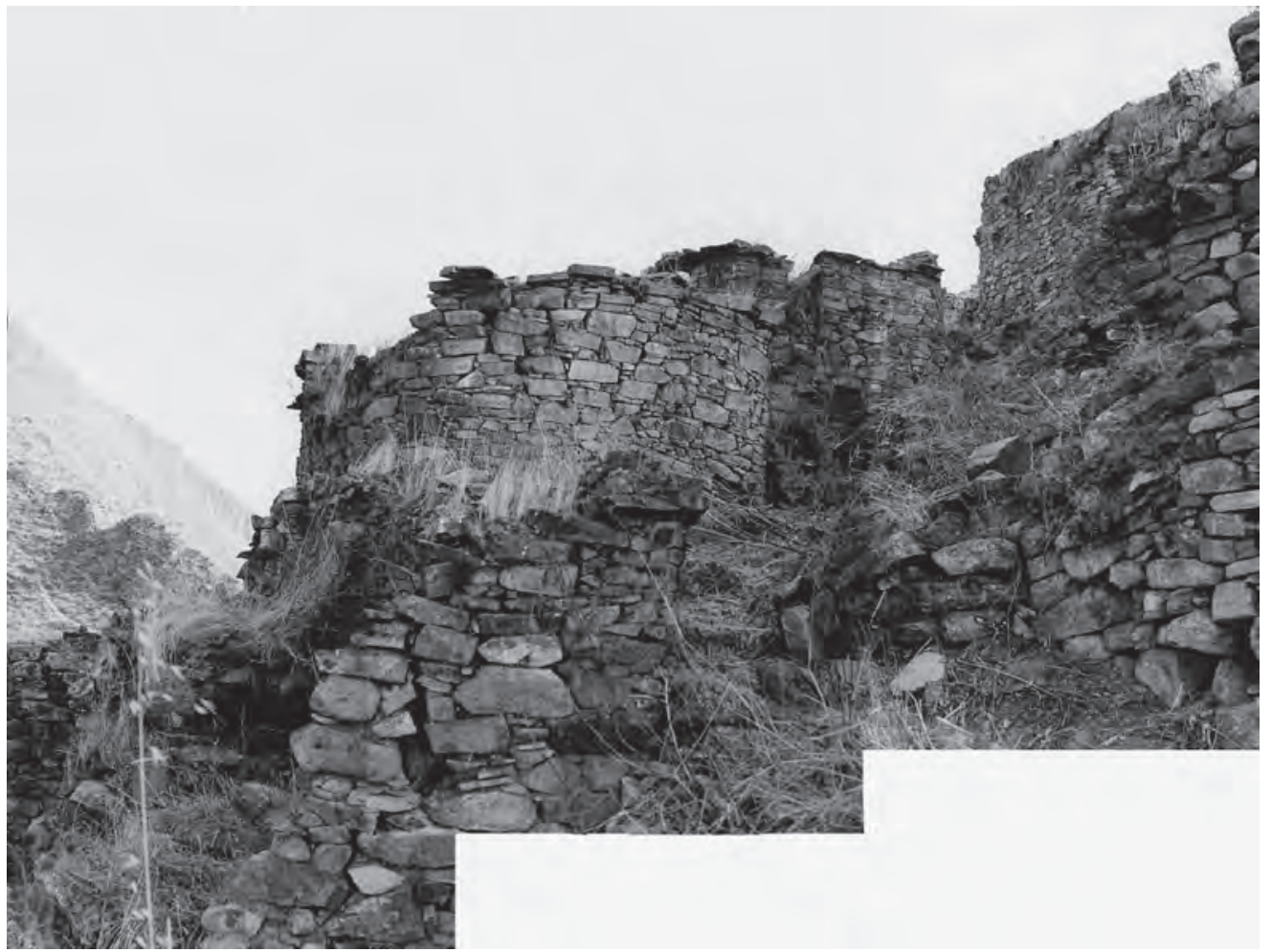

Figura 5: Espacios funcionales al interior de la topografía accidentada. Fotografía M. Luján.

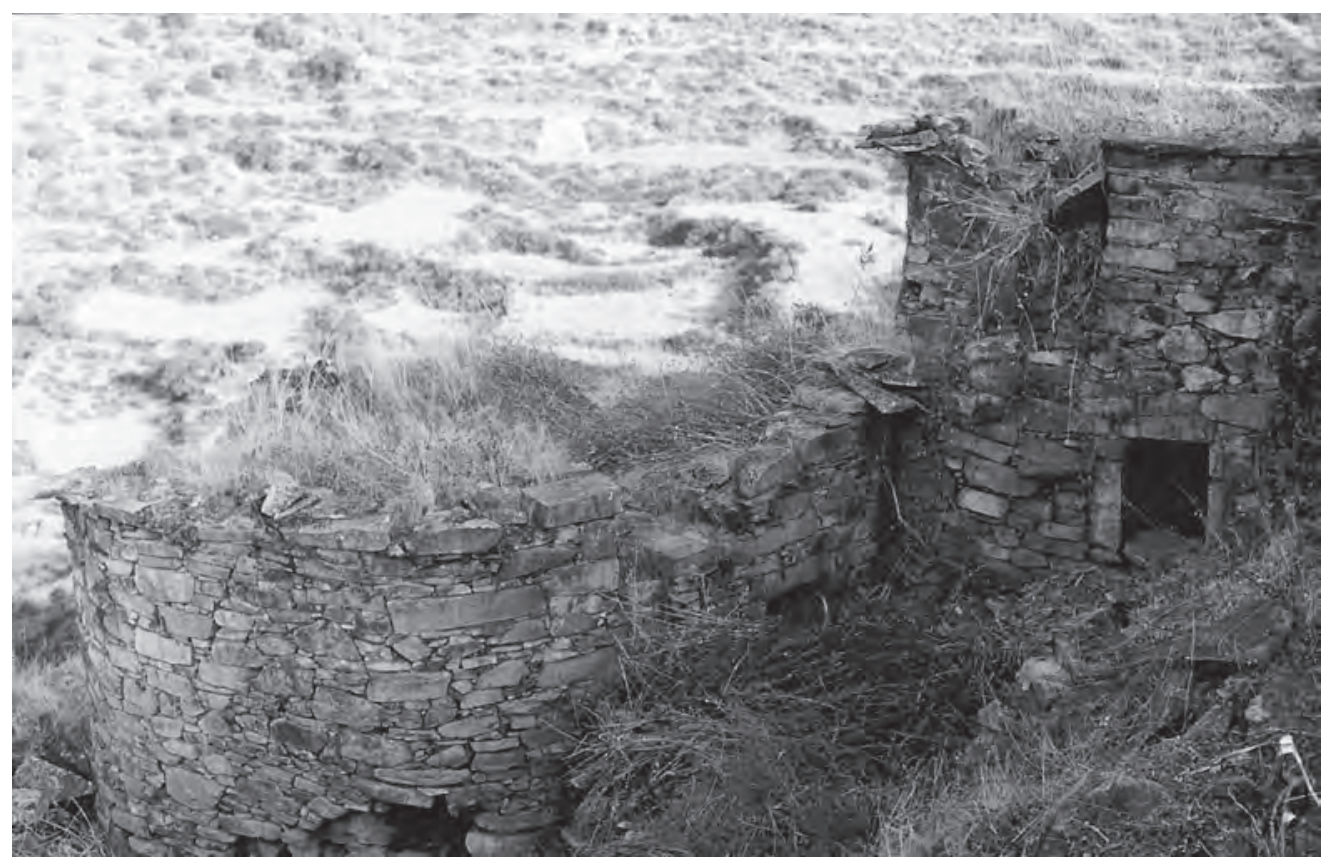

Figura 6: Arquitectura de las unidades familiares. Fotografía M. Luján. 
pacio intramuro y extramuro, modelo recreado por asentamientos pequeños de menor jerarquía como Huanchos, pequeña comunidad que habría albergado a una población promedio de 200 a 300 habitantes con unidades familiares que habrían permitido interpolar de 5 a 15 habitantes dependiendo del espacio interior (Fig. 3).

Asentamiento que no solo estuvo sujeto a la geomorfología o topografía del área, sino también al espacio intramuro que fue adaptado a espacios accidentados a través de terrazas, que fueron transformados en áreas adecuadas para diferentes funciones, sean estos pasajes, patios y recintos (Fig. 6) aprovechando al máximo el uso de los espacios. Adquiriendo de esta manera múltiples beneficios desde un carácter doméstico relacionado directamente con su economía, depósitos y cámaras para almacenamiento (Fig. 7) vinculados a ofrendas establecido en base a hallazgos asociados de manera directa con estos espacios (Fig. 8).
En este sentido, las unidades de residencia en Huanchos, presentan espacios definidos a través de una disposición de elementos arquitectónicos al interior de la unidad domestica, resultado de la morfología y disposición de c. 51 edificios con uso diverso -domestico, funerario, almacenamiento, etc. - - Las cuales presentan en sus lados laterales características arquitectónicas de menor dimensión (Fig.3).

Hacia el lado Oeste de los recintos se observaron ductos de ventilación o chimenea en un $35 \%$ del total de las unidades, hacia el lado Este presentaban dos niveles de cámara en un 50\% del total de los edificios compuestas por 2 cámaras (Fig. 7) pequeñas al interior de cada nivel, compartidos por un muro de cortina, patrón que funcionó también en los sitos de Queca y Viscas, asentamientos que faldean al cerro Lucana o Cahuara «Apu o Camaquen» tutelar de estos asentamientos.

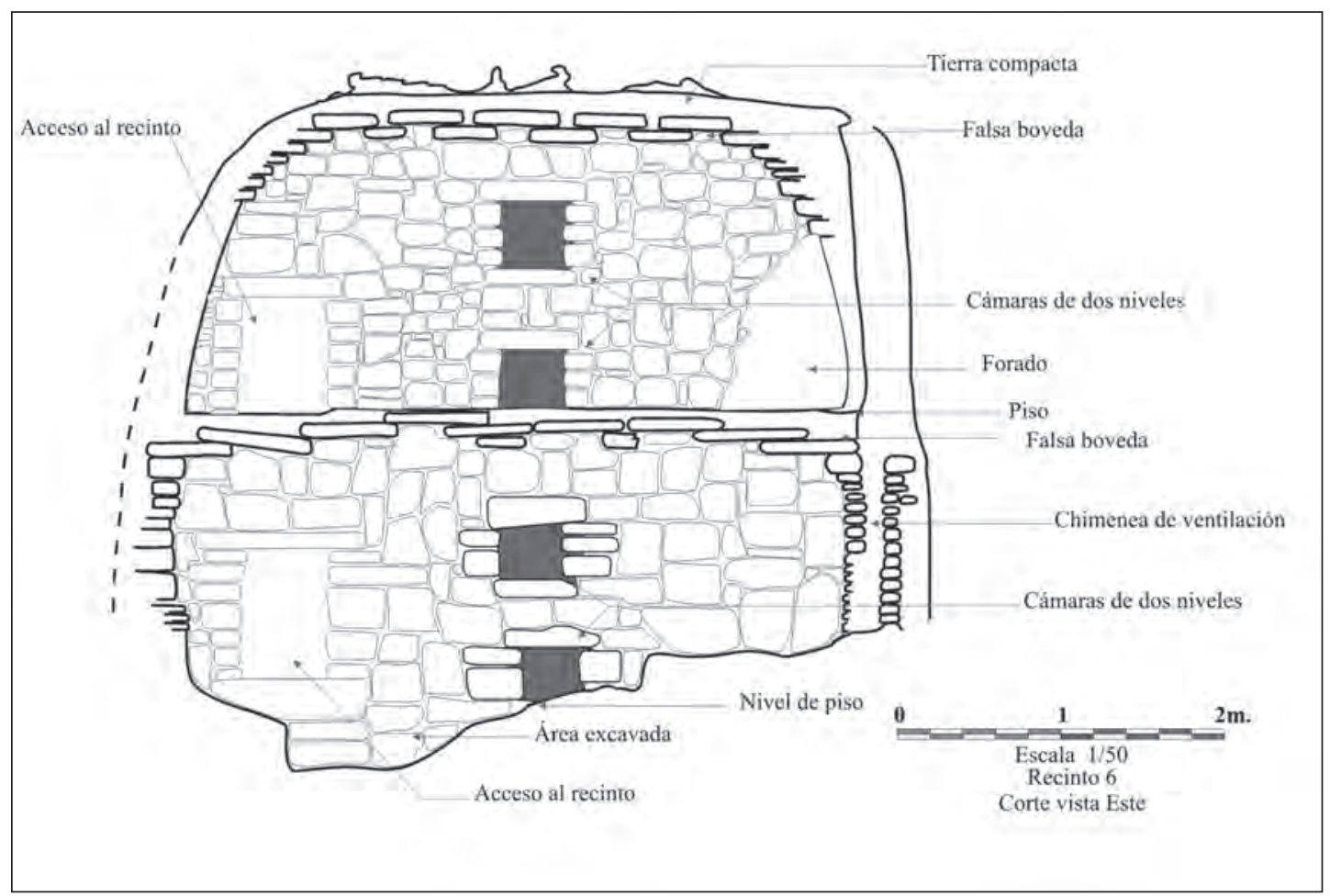

Figura 7: Corte de transversal del R6. Dibujo M. Luján. 
Por lo tanto, la arquitectura de los sitios de la sierra de Lima, en la Cuenca Alta del Chillón, recalan en un optimo grado de conocimiento y manejo en la técnica constructiva en espacios geográficos agrestes, edificadas a través de plantas irregulares de carácter ovalado con acabado propio de la zona, en la que predominará el techo en falsa bóveda (Fig. 7) con uso para actividades domesticas, la cual no solo formo parte de la cuenca alta del Chillón, sino también de las cuencas altas del Chancay, Cañete, etc. Recurso formal y estético que contribuyó a definir uno de los rasgos más sobresalientes de la arquitectura de la sierra de Lima específicamente «Canta».

Elementos como cornisas, aleros voladizos, escaleras empotradas que conducen a espacios abiertos o terrazas que conjugan asimismo en un trabajo planificado entre el arte constructivo y el manejo del espacio, dándole un acabado uniforme en la parte exterior del recinto, donde la piedra canteada muestra su mejor acabado y las unidades de vivienda cumplen de esta manera diversas funciones, desde usos domésticos propios para actividades de una familia o como probables espacios de almacenamiento o despensa en las cámaras superiores y posiblemente funerarias en las cámaras inferiores.

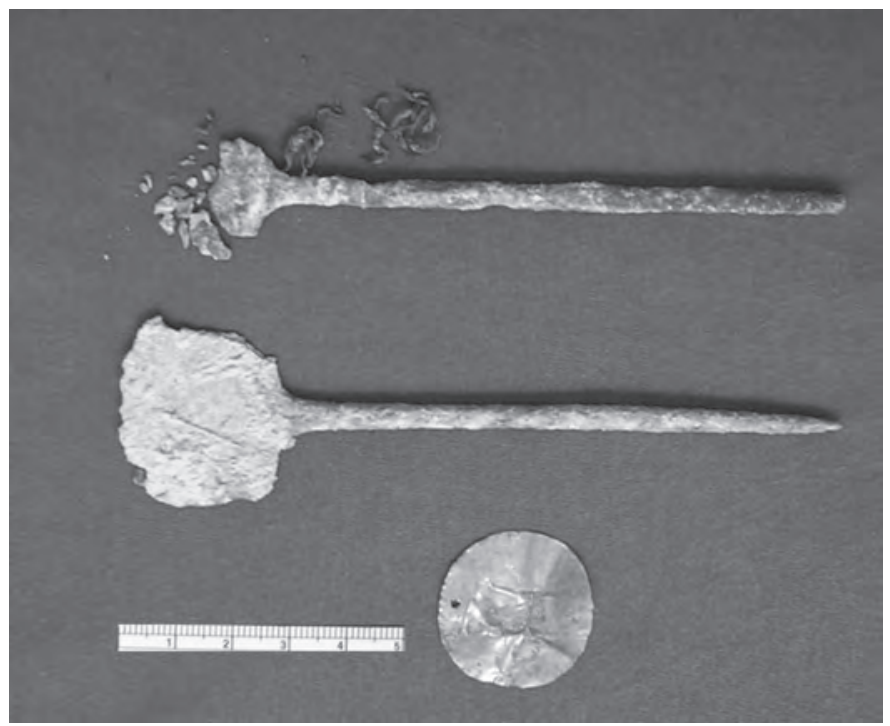

Por lo que, la tecnología desarrollada para los sitios de la parte alta del Chillón parecen presentar de esta manera una mayor complejidad al interior de las unidades de vivienda que las unidades residenciales de sitios como los Astos, wankas, Chupaichus, Chinchaicochas, etc. cuyas edificaciones muestran simplicidad y no dieron lugar a refinamientos arquitectónicos como lo sugiere Thompson (1972), Lavalleé, Julien (1983) y Bonnier (1997).

Durante las excavaciones en la temporada de 1998, se pudo identificar al interior de los edificios gran cantidad de material alfarero de carácter doméstico asociados a bruñidores, óseos de animal aún no identificados dentro de su especie, moluscos, piruros, batanes, manos de moler, etc., relacionados con periodo Intermedio Tardío y Horizonte Tardío, lo que indicaría fuerte ocupación doméstica en los espacios interiores. Otros elementos asociados fueron óseos humanos de entierros secundarios relacionados a concepciones de convivencia con sus ancestros, por último un elemento significativo que estaría asociado dentro de la concepción religiosa son los hallazgos de ofrendas encontradas en pequeños hoyos de forma circular compuesto por dos tupos de cobre y una lamina de oro asociadas a las cámaras (Luján y Sánchez 1999) (Fig. 8).

La solidez constructiva de estas unidades familiares guardan de esta manera un patrón que se refleja no solo en asociación sino entre espacio e individuos, complementado a un espaciomístico religioso representado por el cerro Cahuara.

Es obvio entonces que bajo esta naturaleza, y con las datos obtenidos se pueda identificar al asentamiento de Huanchos con un conjunto de características antagónicas desde un hábitat irregular, en las que se conjugan espacios claramente definidos que habrían estado funcionando directamente sectores I y II (Fig. 3)

Figura 8: Ofrendas halladas al interior de un Recinto. Fotografía M. Luján. 
bajo el control de un jefe comunal en relación directa con el ayllu principal de Viscas, sitio en la cual habrían existido espacios restringidos como son los recintos de la parte alta donde cada unidad familiar habría desarrollado múltiples actividades sin dejar de olvidar fue el espacio administrativo y estaría relacionados directamente a la clase dirigente "Jefe Local" y el grupo de personas que laboraban directamente en todo el área.

Por lo tanto muchos elementos al interior de este sistema sirven de sustento y apoyo para explicar nuestra área de investigación, en la cual el desarrollo asimétrico del sitio se asoció funcionalmente con todo el espacio paisajístico donde se ubican los tres sitios -Viscas, Quecamarca y Huachosmarca-.

\section{CONClusiones}

Desde un análisis espacial intramuro del sitio, compuesto por unidades familiares a través de un conjunto de residencias de carácter doméstico, laboral, depósitos y posiblemente funerario, se podría establecer no solo actividades domesticas sino de convivencia con sus ancestros a través de entierros secundarios y posibles entierros primarios en las cámaras inferiores. El espacio extramuro estuvo sujeto a un sistema de terrazas productivas que llevaron a crear relaciones de productividad y espacio sagrado vinculado al cerro Lucana o Cahuara apu tutelar de los sitios de Viscas, Queca y Huanchos.

En este sentido el crecimiento asimétrico manejado para este asentamiento responde a un patrón típico de sitios de altura en la sierra de Lima, donde se conjugó el espacio como actividad económico, social y religioso, en la cual la arquitectura de los recintos parecen confluir en un misticismo religioso entre $a p u$ y unidad residencial, sitio que posiblemente marque principios de organización comunal en sus diversos aspectos en estas poblaciones de la parte Alta del Chillón. El análisis del manejo del espacio entonces está relacionado con sus vecinos para el Período del Intermedio Tardío en la sierra de Lima, presentan entonces paralelismos de desa- rrollo y crecimiento en diferente orden sea de carácter vertical y horizontal entre los 2400 a los 4000 metros sobre el nivel del mar.

Finalmente, pareciera que toda vinculación político-económico de carácter vertical no fue un único modelo de interacción entre estos grupos sociales, aún cuando estas funcionaron entre pueblos instalados en zonas bajas (Yungas) y altas del Chillón (Quechuas), naturaleza que parece haberse dado también a través de interacciones horizontales en la sierra de Lima, área en la cual parecieran no existir límites culturales que aún no han sido muy bien definidas, la que se ve recreado en la cultura material, economía, crecimientos asimétricos en los asentamientos, así como concepción religiosa integradora de grupos de poblaciones diversificadas por un regionalismo sin demarcación territorial durante el Periodo Intermedio Tardío.

\section{Agradecimientos}

Hago extensivo mi agradecimiento a un gran amigo y colega con quien compartimos el desarrollo de este trabajo de investigación durante mucho tiempo a Luis Sánchez Palomino.

\section{Bibliografía \\ Agurto, Santiago \\ 1989 "Los kullpis de Cantamarca". Ingeniero Civil № 63: Lima. \\ Ávila, Francisco de \\ 2009 "Dioses y Hombres de Huarochiri". Tra- ducción J. M. Arguedas. Universidad Antonio Ruiz de Montoya. \\ Bonier, Elizabeth \\ 1997 "Morfología del espacio aldeano y su ex- presión cultural en los Andes Centrales". Arquitectura y Civilización en los andes Prehispánicos. Archeologica Peruana 2: 28-41Sociedad Arqueológica Peruano- Alemana, Reiss Museum Mannheim, \\ Cornejo, Miguel \\ 2000 "La Nación Ischma y la provincia Inca de Pachacamac". Arqueológicas No. 24: 149-173 M.N.A.A.H. Lima, Perú.}


Dillehay, Tom

1976 "Copetition and copertation in a prehispanic multi-ethnic system in the Central Andes". Ph. D Dissertation, Departament of Anthropology, University of Texas, Austin.

1977 "Un estudio de almacenamiento, redistribución y dualismo socio-político prehispánico en la Chuapiyunga del valle del Chillón". Cuadernos del CONUP. № 24 - 25: 25-37. Lima.

1987 "Estrategias políticas y económicas de las etnias locales del valle del Chillón, durante el Periodo Prehispánico". Revista Andina Año 5, № 2: 407-455. Cuzco.

Dillehay, Tom; Patricia Netherly

1998 "Introducción" Las Fronteras del Estado inca. Fundación Alexander Von Humboldt y Ediciones. ABYA-AYALA. 3-33 Ecuador.

Earle, Timothy, Terence D’Altroy, Katherine LeBlanc

1978 "Arqueología regional de los periodos prehispánicos tardíos en el Mantaro". Actas del III Congreso Peruano del Hombre y la Cultura Andina. 641-672

Farfán, Carlos

1988 "Informe preliminar de los trabajos arqueológicos en Cantamarca”. Actas y trabajos del VI Congreso Peruano del Hombre y la Cultura Andina, Editorial Francisco Iriarte B. Vol 3: 147-167. Lima

1995 "Asentamientos prehispánicos en la cuenca Alta del Chillón". Gaceta arqueológica Andina № 24. Pp. 31-61 Lima.

Favre, Henrry

1983 "Introducción”. ASTO: Curacazgo Prehispánico de Los Andes Centrales IEP Lima.

Huertas, Lorenzo

1981 "La religión en una sociedad rural Andina (Siglo XVII)". Edit. Universidad $\mathrm{Na}$ cional de San Cristóbal de Huamanga. Ayacucho- Perú.

Kaulicke, Peter

1974- 1975 "Reflexiones sobre la arqueología de la sierra de Lima". Boletín de Seminario Arqueología IRA. No. 15 - 16, No.96: 29-60 Instituto Riva Agüero. PUCP. Lima.
Lavalée, Daniéle y Michéle Julien

1983 “ASTO: Curacazgo Prehispánico de Los Andes Centrales". IEP Lima.

Luján, Milton

2009 "Informe Proyecto de Investigación Arqueológico Huacosmarca Cuenca Alta del Chillón, Canta”. Presentado al INC. Lima.

Luján, Milton y Luis Sánchez

1999 "Informe de las investigaciones arqueológicas en el sito de Huanchormarca, comunidad de San Lorenzo, distrito Lachaqui, Canta". presentado al INC Lima.

Marcus, Joyce y Jorge Silva

1988 "The Chillon valley coca lands: archeologiacal back ground ecological context". Estudies in Latin American Etnohistory and archelogy. Memoirs of the museum of anthropology. Universty of Michigan No 21vol IV: 1-52

Miniesterio de Fomento

1881 "Relaciones Geográficas de Indias" Perú Tomo I. Madrid - España

Murra, John V.

1975 "Formaciones Económicas y Políticas del Mundo Andino". IE P. Lima. Perú.

1977 "La Organización Económica del Estado Inca”. Editorial Siglo XXI México.

2009 "El Mundo Andino población, medio ambiente y economía" Fondo Editorial de la Pontificia Universidad Católica e Instituto de Estudios Andinos.

Netherly, Patricia

1998 "El reino Chimor y el Tawantinsuyo". La Frontera del Estado inca. Compiladores T. Dillehay y P. Netherly. Fundación Alexander V. Humboldt, Abya Yala. 85-105 Quito.

Parsons, Jeffrey y Ramiro Matos

1977 "Asentamientos prehispánicos en el Mantaro - Perú". Informe Preliminar Tercer congreso Peruano del Hombre y la Cultura Andina. Tomo II.

Raffino, Rodolfo

1991 "Poblaciones Indígenas en Argentina: Urbanismo y Proceso Social Precolombino" T.E.A. Buenos Aires. Argentina 
Rostworowski, María

1973 "Las etnias del valle del Chillón". Revista del Museo Nacional. Tomo 38: 250-314.

1978 "Los Señoríos Indígenas de Lima y Canta". IEP. Lima.

1986 "Estructuras Andinas del Poder: Ideología, Religión y Política”. IEP. Lima

Schjellrup, Inge

1992 "Los Andes de La Región de Patrones de Asentamiento en Las Faldas Orientales de Chachapoyas". Estudios de Antropología Peruana. Edit. Fomciencias, Editor: Duccio Bonavia. 355-374 Lima, Perú.

Silva, Jorge

1996 "Prehistoric settlement patterns in the Chillon river valley, Perú”. A dissertation submitted in partial fulfillment of the requirements for the degree of doctor of Philosophy Anthrolopology, in the University of Michigan.
Thompson, Donald E.

1972 "Etnias y Grupos Locales Tardíos. Pueblos y culturas de la sierra del Perú". Cerro de Pasco Cooper Corporation. 66-75 Lima. Perú.

Villar Córdoba, Pedro

1935 "Arqueología del Departamento de Lima: Las culturas prehispánicas del departamento de Lima". Editorial Atusparia 423 - 2da Edición 1982. 